\title{
Determinación de anticuerpos contra patógenos virales y bacterianos seleccionados en la población de cerdos silvestres (Sus scrofa) de la Reserva Natural Bahía Samborombón, Argentina
}

\section{Determination of antibodies against selected viral and bacterial pathogens in the feral pigs (Sus scrofa) population of the Bahía Samborombón Natural Reserve, Argentina}

\author{
Carpinetti $\mathrm{B}^{1,3^{*}}$, Castresana $\mathbf{G}^{3}$, Rojas $\mathrm{P}^{3}$, Grant $\mathrm{J}^{2}$, Marcos $\mathrm{A}^{2}$, Monterubbianesi $\mathbf{M}^{2}$, \\ Sanguinetti $\mathrm{HR}^{4}$, Serena $\mathbf{M S}^{5,6}$, Echeverría MG ${ }^{5,6}$, Garciarena $\mathbf{M}^{1}$, Aleksa $A^{1}$
}

\begin{abstract}
${ }^{1}$ Cátedra de Ecología y Recursos Naturales, Universidad Nacional Arturo Jauretche; 2Dirección Nacional de Sanidad Animal, Servicio Nacional de Sanidad y Calidad Agroalimentaria (SENASA); ${ }^{3}$ Dirección de Áreas Naturales Protegidas, Organismo Provincial para el Desarrollo Sostenible de la Provincia de Buenos Aires; ${ }^{4}$ Dirección de Laboratorio Animal, SENASA; ${ }^{5}$ Consejo Nacional de Investigaciones Científicas y Técnicas (CONICET); ' 'Laboratorio de Virología, Facultad de Ciencias Veterinarias, Universidad Nacional de La Plata.

*Correo electrónico del autor: brunoelcarpincho@hotmail.com
\end{abstract}

\begin{abstract}
Resumen: Los cerdos silvestres (Sus scrofa) descienden de cruzamientos entre cerdos domésticos liberados durante la colonización con jabalíes salvajes euroasiáticos, liberados con propósitos cinegéticos. Son invasivos y su coexistencia con especies domésticas implica riesgos sanitarios. Argentina es considerada libre de fiebre aftosa (FA), peste porcina clásica (PPC) y africana (PPA) y síndrome reproductivo y respiratorio porcino (PRRS). La enfermedad de Aujeszky (EA) y la leptospirosis son endémicas en ciertas áreas del país. El objetivo fue evaluar la presencia de ciertas enfermedades zoonóticas y/o de importancia para la producción animal y la conservación de la biodiversidad en cerdos silvestres de la Bahía de Samborombón. Se capturaron 118 animales. Se tomaron muestras de suero, tonsilas, músculo, intestino delgado, linfonódulos, entre otras. Se estudió la presencia de anticuerpos contra Brucella spp., coronavirus respiratorio porcino, virus de la estomatitis vesicular, de la FA, de la gastroenteritis transmisible porcina (TGEV), de la PPC, PPA, EA, PRRS y Leptospira spp. Se realizaron análisis bacteriológicos para Mycobacterium spp. Los resultados ratificaron la ausencia de las enfermedades exóticas e indicaron que $36 \%$ de los animales presentó anticuerpos contra Leptospira interrogans serovar pomona y $62,5 \%$ contra el virus de la EA. Estos resultados remarcan la importancia del monitoreo de la interfase productiva/silvestre en función de la salud pública, producción animal y conservación de la biodiversidad.
\end{abstract}

Palabras clave: cerdos silvestres, Sus scrofa, Bahía Samborombón, zoonosis

\begin{abstract}
Overlapping between wildlife and domestic species implies the risk of disease transmission. In Argentina, the wild pig (Sus scrofa) is a widespread invasive species. Sus scrofa wild populations are descendants from domestic pigs released during colonisation and Eurasian wild boars released for sport hunting purposes. Argentina is considered free from foot-and-mouth disease (FMD), classical swine fever (CSF), African swine fever (ASF) and porcine reproductive and respiratory syndrome (PRRS). Aujeszky disease (AD) and leptospirosis are considered endemic in certain areas of the country. The aim of this study was to evaluate the presence of some zoonotic and productive diseases in the wild pig population of Bahía Samborombón, Argentina. A total of 118 animals were captured. Samples consisted on serum, tonsils, muscle, small intestine and lymph nodes. Detection of antibodies for Brucella spp, porcine respiratory coronavirus (PRCV), vesicular stomatitis virus (VSV), foot-and-mouth disease virus (FMDV), porcine transmissible gastroenteritis virus (TGEV), Leptospira spp, CSF virus, ASF virus, AD virus and PRRS virus, as well as bacteriological analysis to detect Mycobacterium spp., were performed. Preliminary results indicate that $36 \%$ and $62.5 \%$ of animals had antibodies against Leptospira interrogans serovar Pomona, and ADV, respectively. These results highlight the importance of closer monitoring on the wildlife/livestock interface for public health, animal production and biodiversity conservation.
\end{abstract}

Keywords: wild boar, Sus scrofa, Bahia Samborombón, zoonosis

Fecha de recepción: 14/05/16

Analecta Vet 2017; 37 (1): 21 - 27

Fecha de aprobación: 18/10/16

Impresa ISSN 0365514-8 Electrónica ISSN 1514-2590 


\section{Introducción}

El jabalí europeo y una amplia variedad de morfotipos de cerdos domésticos asilvestrados (Sus scrofa) se han naturalizado y se encuentran ampliamente distribuidos en Argentina (Merino \& Carpinetti, 2003). Algunas de estas poblaciones silvestres son descendientes de cerdos domésticos liberados durante la colonización española (Crosby, 1986; Navas 1987), mientras que otras se originaron a partir de ejemplares de jabalí euroasiático puro deliberadamente introducidos durante la primera década del siglo xx con propósitos cinegéticos (Daciuk, 1978; Navas, 1987). La hibridación entre poblaciones silvestres y cerdos domésticos criados a campo también es común, resultando en un patrón muy confuso de distribución e interrelaciones entre las variedades domésticas, silvestres y formas híbridas (Merino \& Carpinetti, 2003).

Las poblaciones silvestres de Sus scrofa pueden actuar como reservorios de enfermedades infecciosas para los animales domésticos y la fauna autóctona, como así también ser fuente potencial de enfermedades zoonóticas (Al Dahouk et al., 2005; Carpinetti et al., 2014; Meng et al., 2009). De esta manera, las interacciones entre especies pueden potencialmente resultar en la diseminación de estos patógenos (Vengust et al., 2006). Además, los cambios en el ambiente tienden a favorecer el contacto entre la fauna silvestre, los animales de producción y el ser humano, y esto juega un rol fundamental en el papel cada vez mayor de la vida silvestre en la aparición de enfermedades animales y humanas (Jones et al., 2008).

Entre las enfermedades infecciosas más frecuentemente citadas en poblaciones de cerdos silvestres se encuentran la brucelosis porcina (Al Dahouk et al., 2005; Ebani et al., 2003; Gresham et al., 2002; van der Leek et al., 1993), leptospirosis (Ebani et al., 2003; Mason et al., 1998), enfermedad de Aujeszky -EA- (Corn et al., 2004; Gresham et al., 2002; Lari et al., 2006; Müller et al., 1998; Zupancic et al., 2002), peste porcina clásica -PPC- (Kern et al., 1999; Zupancic et al., 2002) y tuberculosis (Naranjo et al., 2008; Nishi et al., 2006).

Argentina está internacionalmente reconocida como país libre de fiebre aftosa (FA), peste porcina clásica (PPC), peste porcina africana (PPA) y síndrome reproductivo y respiratorio porcino (PRRS). En general, la vigilancia epidemiológica llevada a cabo por los organismos oficiales de control está concentrada principalmente en el muestreo de animales domésticos, aportando escasa información sobre la presencia y prevalencia de agentes infecciosos en poblaciones de animales silvestres.

La Bahía Samborombón es el humedal mixohalino más extenso de Argentina. Se localiza en la costa de la provincia de Buenos Aires, en el último tramo del estuario del Río de la Plata y se extiende desde Punta Piedras ( $\left.35^{\circ} 27^{\prime} \mathrm{S} ; 5^{\circ} 45^{\prime} \mathrm{W}\right)$ hasta Punta Rasa (36²2'S; 56³5'W).

La vegetación del área es un mosaico con presencia de bancos de conchillas donde crecen bosques de tala (Celtis tala) y determinado por factores tales como la duración y extensión de las inundaciones de agua dulce o estuariales y por las características del suelo. Dicha región es un área protegida ${ }^{1}$ y alberga una gran biodiversidad de especies nativas, algunas de ellas en peligro de extinción, como el venado de las pampas (Ozoteceros bezoarticus celer) (Merino \& Carpinetti 2003), especies introducidas como el ciervo axis (Axis axis), perros asilvestrados (Canis familiaris) y cerdos silvestres o cimarrones (Sus scrofa). Además, coexisten en el área asentamientos humanos dedicados a la explotación ganadera (Carpinetti et al., 2014).

Se ha sugerido que el incremento de la población de cerdos silvestres (Sus scrofa) es una de las causas actuales más importantes que afectan la recuperación del venado de las pampas en la Bahía (Carpinetti 1998; Merino \& Carpinetti 2003). Algunos estudios preliminares sugieren que, en Samborombón, el incremento de cerdos silvestres podría afectar la supervivencia del venado a largo plazo (GiménezDixon 1991; Merino \& Carpinetti 2003), ya que se ha señalado que los cerdos silvestres pueden competir y/o excluir espacialmente a esta especie amenazada, además de no descartarse la predación sobre crías (Merino et al., 1993; Carpinetti 1998). También esta especie afecta otros valores de conservación del área y produce un significativo impacto sobre la biota nativa.

En los últimos años, Argentina ha registrado un fuerte y sostenido crecimiento de la producción y del consumo de carne porcina. Con el aumento en la cantidad y calidad de los establecimientos de producción porcina en el país, aumentan las exigencias de elevar sus niveles sanitarios para garantizar productividad y aspirar a mercados internacionales. En este escenario, la vigilancia epidemiológica de las poblaciones silvestres de cerdos adquiere especial relevancia.

En este sentido, el objetivo de este estudio fue determinar la presencia de anticuerpos contra una serie de patógenos seleccionados por su importancia para la salud humana, la producción animal y/o la conservación de la fauna silvestre, en la población de cerdos silvestres de la Reserva Natural Bahía Samborombón.

\footnotetext{
Reserva Natural Bahía Samborombón, Ley 12016/97. Categoría de manejo: Reserva Natural Integral, Reserva Natural de Objetivo Definido y Refugio de Vida Silvestre.
} 


\section{Materiales y métodos}

Entre enero de 2013 y abril de 2015, dependiendo de las condiciones climáticas y de la disponibilidad técnica y logística de los guardaparques de la reserva natural, se capturaron cerdos silvestres utilizando distintas técnicas en el marco de las tareas rutinarias de control de esta especie introducida en el área protegida (Figura 1). Con el de fin de evitar riesgos sanitarios, los guardaparques fueron instruidos por profesionales del Servicio Nacional de Sanidad y Calidad Agroalimentaria (SENASA) con respecto a las normas de seguridad durante la apertura de cadáveres.

La extracción y el acondicionamiento de muestras de los cerdos capturados se realizaron de acuerdo con el protocolo elaborado por personal técnico del SENASA. Se colectaron muestras de tonsilas, linfonódulos, hígado, riñones, intestino delgado, músculos (diafragma y músculo masetero) y sangre para la obtención de suero para serología. Las muestras fueron remitidas para su análisis al SENASA, Dirección General de Laboratorio y Control Técnico (DILAB) ubicado en la localidad de Martínez, provincia de Buenos Aires.

Para la determinación de anticuerpos contra PPC, PPA, EA, virus de la gastroenteritis transmisible porcina (TGE) y PRRS se utilizaron kits de ELISA comerciales: IDEXX CSFV Ab Test, Ingezim PPA, IDEXX PRVIADV gl Ab Test, Ingezim Corona Diferencial 2.0 e IDEXX PRRS X3 Ab Test, respectivamente. Para el diagnóstico de FA se evaluó la presencia de anticuerpos contra el antígeno asociado a la infección (VIA) a través de la técnica de inmunodifusión en gel de agar (IDGA). Asimismo, se realizó la búsqueda de antígeno viral para PPC mediante la técnica de inmunofluorescencia directa (IFD) a partir de muestras de tonsilas.

En el caso de los patógenos bacterianos, la detección de anticuerpos contra Brucella spp. se realizó mediante la técnica de aglutinación con antígeno buferado en placa (BPA) y, como técnica confirmatoria, se utilizó la prueba de seroaglutinación lenta en tubo (SAT). En el caso de Leptospira spp. se realizó la microaglutinación en tubo (MAT). Para el diagnóstico de tuberculosis los linfonódulos fueron descontaminados por la técnica de Petroff y sembrados en medios específicos para micobacterias (Mycobacterium spp.), Lowestein-Jensen y Stonebrink, durante, al menos, 90 días y en caso de existir desarrollo bacteriano compatible con bacilos ácido-alcohol resistentes se tipificaron mediante métodos moleculares.

Todas las técnicas mencionadas son las recomendadas en el "Manual de las Pruebas de Diagnóstico y de Vacunas para los Animales Terrestres" de la Organización Mundial de Sanidad Animal (OIE, 2015).

\section{Resultados}

En el período comprendido entre enero de 2013 y abril de 2015 se capturaron 118 animales y se realizaron un total de 1325 determinaciones (Tabla 1). Las enfermedades analizadas como FA, PPC, PPA, PRRS y TGE arrojaron resultados negativos en su totalidad. Con respecto a Leptospira spp., se detectó un 36\% (30/83) de serología positiva para $L$. interrogans serovar pomona. La mayoría de los títulos obtenidos fueron bajos (menos de $1 / 200)$. Con respecto a la EA, se registró un $62,5 \%$ (65/104) de animales positivos. Asimismo, se detectaron dos casos positivos de Brucella spp. mediante la técnica de tamiz, aunque sólo uno fue confirmado con la técnica de SAT sobre un total de 96 animales analizados.

\section{Discusión}

Los resultados obtenidos ratifican la ausencia en la población bajo estudio de enfermedades consideradas exóticas en la Argentina, como PPC, PPA y PRRS, encontrándose ellas bajo plan de vigilancia oficial.

El último foco de PPC fue registrado en el año 1999. Luego de la aplicación de la Resolución $\mathrm{N}^{\circ}$ $834 / 2002$ se logró finalmente erradicar la enfermedad $y$, en el año 2005, siguiendo las recomendaciones de la OIE, la República Argentina se autodeclaró "libre de peste porcina clásica" mediante la Resolución SAGPYA No $343 / 2005$.

A partir de ese año, y hasta la actualidad, el SENASA lleva a cabo actividades de vigilancia epidemiológica con el objetivo de detectar en forma precoz la reaparición de la enfermedad y recolectar información para sustentar o documentar el estatus de país libre. Estas actividades se llevan a cabo junto al monitoreo de otras dos enfermedades virales, como son el síndrome respiratorio reproductivo porcino y la peste porcina africana. Estas enfermedades nunca han sido diagnosticadas en la República Argentina, por lo que son consideradas exóticas por Resolución del SENASA 422/2003.

Las actividades de vigilancia que se llevan a cabo para PPC, PPA y PRRS se basan en la prevención debido al impacto económico y social que conllevaría la ocurrencia de un foco.

Con respecto a las enfermedades consideradas endémicas, el porcentaje de cerdos silvestres con anticuerpos contra la EA podría indicar el contacto entre los mismos y los cerdos domésticos, específicamente con la población de cerdos domésticos de traspatio o pequeñas producciones al aire libre, siendo éste el estrato productivo con mayor prevalencia de la enfermedad ${ }^{2}$. 2Sobre la base de un muestreo serológico nacional realizado por el SENASA
en el año 2010 para determinar la prevalencia de cerdos infectados por el virus
de la EA, en el marco del Programa Nacional de Control y Erradicación de la
Enfermedad de Aujeszky aprobado por Resolución ex SAGPyA N ${ }^{\circ}$ 474/2009. 


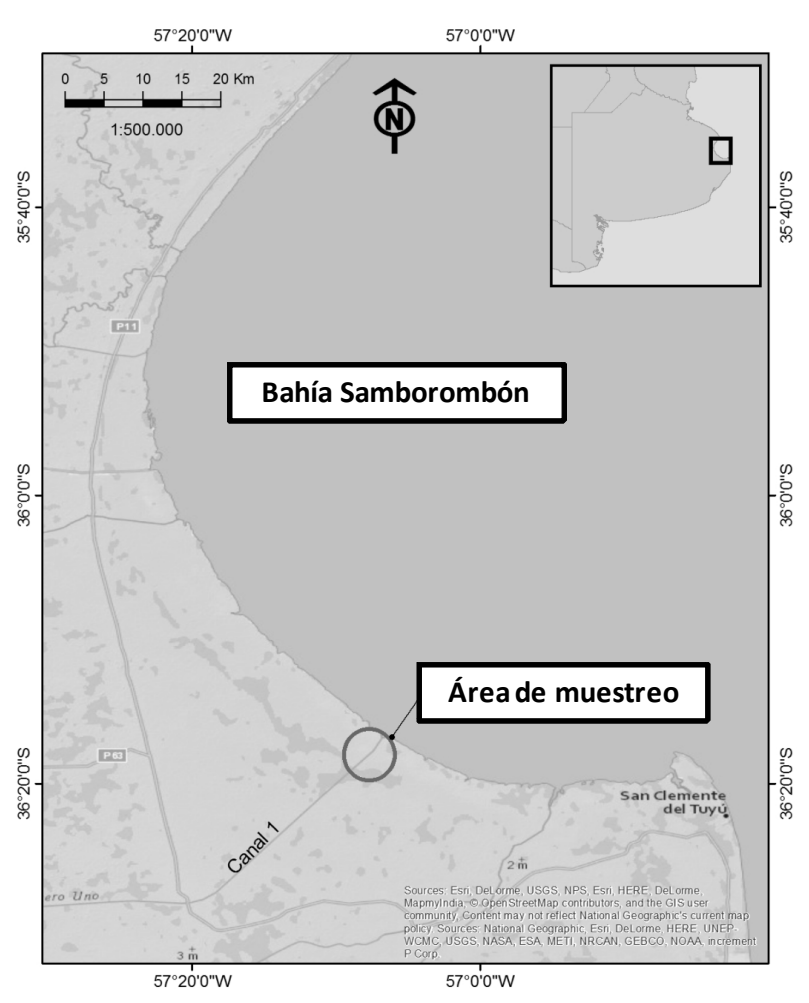

Figura 1. Área de muestreo en Bahía Samborombón, provincia de Buenos Aires.

En Brasil, en la región del Pantanal se realizó un estudio que determinó una prevalencia del $47,3 \%$ de positivos a EA (Paes et al., 2013).

Durante 2013-2014 nuestro grupo ha realizado el primer estudio que analizó el estado sanitario de cerdos salvajes con respecto a las principales virosis que afectan a los porcinos. Teniendo en cuenta que estos animales representan una amenaza para la salud del hombre y de los animales domésticos, y que el impacto económico de las enfermedades que los afectan es muy importante, deben ser contemplados dentro de los planes sanitarios llevados a cabo por las distintas agencias del Estado. En relación con los resultados obtenidos para el caso de la EA (casi un $40 \%$ de positivos), los mismos revelan la posible presencia de cerdos salvajes con enfermedad en estado de latencia, como así también una posible circulación viral en dicha población de animales, al detectarse títulos serológicos elevados. Estos resultados provenían de muestreos de Bahía Samborombón y de cotos de caza en Quemú-Quemú (provincia de La Pampa) y Villa Huidobro (provincia de Córdoba). D e esta manera, se prevé continuar trabajando y utilizar los resultados obtenidos para contribuir con la campaña de erradicación de la enfermedad (Serena et al., 2015).

Asimismo, la detección de anticuerpos contra Leptospira interrogans serovar pomona indica que en los ambientes de la Bahía Samborombón existe contacto de los cerdos silvestres (Sus scrofa) con la bacteria, representando un posible riesgo no solo para otros animales silvestres sino también para la salud pública, aún cuando el serovar prevalente en Argentina en cerdos domésticos es Castellonis, seguido por Icterohaemorrhagiae (Petrakovsky et al., 2013 \& 2016). El serovar Pomona está asociado principalmente al ganado bovino y en segundo lugar al ganado porcino (Bharti et al., 2003). En este estudio no fue posible evaluar seroconversión para determinar si se trató de una infección aguda o crónica, ya que los animales fueron capturados y sacrificados. Este mismo serovar ha sido encontrado con mayor frecuencia en Sus scrofa en el área de Berlín, Alemania (Jansen et al., 2007).

Además de su distribución mundial, la leptospirosis es una de las enfermedades con mayor impacto económico a causa de las grandes pérdidas que ocasiona en la ganadería en general, como así también en la producción porcina, relacionadas con abortos, alta mortalidad perinatal, disminución en el tamaño de camada, entre otros (AAVLD, 1994). El cerdo es el mejor ejemplo de un animal portador/excretor, ya que una vez que las leptospiras se localizan en el riñón, el animal se transforma en eliminador de bacterias de por vida, jugando un rol de gran importancia en el ciclo de la enfermedad, semejante al de varias especies de roedores. Dado el importante rol de los cerdos como reservorios en la cadena epidemiológica de la leptospirosis, los hallazgos presentados en este trabajo ponen de manifiesto la relevancia de continuar los estudios de vigilancia de este tipo sobre la población de estos animales y otros mamíferos silvestres.

Desde el punto de vista de la conservación de especies amenazadas de extinción, las medidas destinadas al control de enfermedades, en general, sólo se han puesto en práctica en el contexto de epidemias que han causado repentinas e importantes bajas en la población de especies en riesgo (Hudson et al., 2002). Sin embargo, los patógenos que causan altas tasas de mortalidad no pueden persistir en poblaciones pequeñas, por lo que los patógenos generalistas, como la leptospira, revisten mucho más riesgo para especies como el venado de las pampas que para los dependientes de él como hospedador específico. Es por ello que las evaluaciones del riesgo de la población del venado de las pampas deberían tener en cuenta, no sólo el impacto de patógenos que causan altas mortalidades, sino el de aquellos causantes de enfermedades crónicas y persistentes en ambientes como el de la Bahía Samborombón, y que podrían causar una declinación de la población a través de sus efectos sobre la fecundidad y la natalidad.

Por otra parte, en el marco del plan de vigilancia para la erradicación de la EA en el país, la detección de una mayor cantidad de animales portadores del 
Tabla 1. Enfermedades estudiadas, tipo de muestras y métodos de diagnóstico utilizados.

\begin{tabular}{|c|c|c|c|c|c|c|c|c|c|c|c|c|c|}
\hline & & & 2013 & & & 201 & & & 2015 & & & Total & \\
\hline $\begin{array}{l}\text { Enfermedad y } \\
\text { tipo de análisis }\end{array}$ & Muestra & $\mathrm{n}$ & Pos & $(\%)$ & $\mathrm{n}$ & Pos & $(\%)$ & $\mathrm{n}$ & Pos & $(\%)$ & $n$ & Pos & $\%$ \\
\hline $\begin{array}{l}\text { Aislamiento } \\
\text { bacteriológico* }\end{array}$ & Linfonódulo & 5 & - & - & 28 & 8 & 28,57 & $\mathrm{nr}$ & $\mathrm{nr}$ & $\mathrm{nr}$ & 33 & 8 & 24,24 \\
\hline $\begin{array}{l}\text { Brucella spp.: } \\
\text { BPA }\end{array}$ & Suero & 65 & - & - & 28 & 2 & 7,14 & 13 & - & - & 106 & 2 & 1,89 \\
\hline $\begin{array}{l}\text { PRCV: ELISA } \\
\text { de bloqueo }\end{array}$ & Suero & 43 & - & - & 21 & - & - & 9 & - & - & 73 & - & - \\
\hline VSV: ELISA & Suero & 5 & - & - & 6 & - & - & $\mathrm{nr}$ & $\mathrm{nr}$ & $\mathrm{nr}$ & 11 & - & - \\
\hline FA: VIA & Suero & 65 & - & - & 29 & - & - & 9 & - & - & 103 & - & - \\
\hline $\begin{array}{l}\text { Leptospira } \\
\text { spp.: Aisla- } \\
\text { miento }\end{array}$ & Riñón & 52 & 1 & 1,92 & 22 & - & - & $\mathrm{nr}$ & $\mathrm{nr}$ & $\mathrm{nr}$ & 74 & 1 & 1,35 \\
\hline $\begin{array}{l}\text { Leptospira } \\
\text { spp.: MAT }\end{array}$ & Suero & 50 & 23 & 46,00 & 29 & 7 & 24,14 & 4 & - & - & 83 & 30 & 36,14 \\
\hline $\begin{array}{l}\text { Mycobacterium } \\
\text { spp: Aislamien- } \\
\text { to }\end{array}$ & Linfonódulo & 40 & - & - & $\mathrm{nr}$ & $\mathrm{nr}$ & $\mathrm{nr}$ & $\mathrm{nr}$ & $\mathrm{nr}$ & $\mathrm{nr}$ & 40 & - & - \\
\hline $\begin{array}{l}\text { PPA: ELISA de } \\
\text { bloqueo }\end{array}$ & Suero & 65 & - & - & 21 & - & - & 9 & - & - & 95 & - & - \\
\hline PPC: IFD & $\begin{array}{l}\text { Intestino } \\
\text { delgado }\end{array}$ & 27 & - & - & 37 & - & - & $\mathrm{nr}$ & $\mathrm{nr}$ & $\mathrm{nr}$ & 64 & - & - \\
\hline PPC IFD & Tonsila & 35 & - & - & 45 & - & - & $\mathrm{nr}$ & $\mathrm{nr}$ & $\mathrm{nr}$ & 80 & - & - \\
\hline $\begin{array}{l}\text { PPC: ELISA } \\
\text { IDEXX }\end{array}$ & Suero & 65 & - & - & 29 & - & - & 9 & - & - & 103 & - & - \\
\hline $\begin{array}{l}\text { PRRS: Elisa } \\
\text { indirecto }\end{array}$ & Suero & 65 & - & - & 29 & - & - & 9 & - & - & 103 & - & - \\
\hline $\begin{array}{l}\text { EA: ELISA } \\
\text { IDEXX (ADV } \\
\text { gp1) }\end{array}$ & Suero & 65 & 40 & 61,54 & 29 & 18 & 62,07 & 10 & 7 & 70,00 & 104 & 65 & 62,50 \\
\hline $\begin{array}{l}\text { EA: ELISA } \\
\text { IDEXX (ADV } \\
\text { gp1) }\end{array}$ & $\begin{array}{l}\text { Trasudado } \\
\text { muscular }\end{array}$ & 5 & 1 & 20,00 & 8 & 4 & 50,00 & 8 & 5 & 62,50 & 21 & 10 & 47,62 \\
\hline EA: PCR & Tonsila & $\mathrm{nr}$ & $\mathrm{nr}$ & $\mathrm{nr}$ & 29 & 11 & 37,93 & 9 & - & - & 38 & 11 & 28,95 \\
\hline $\begin{array}{l}\text { TGE: ELISA In- } \\
\text { gezim Corona } \\
\text { Diferencial }\end{array}$ & Suero & 65 & - & - & 29 & - & - & 9 & - & - & 103 & - & - \\
\hline
\end{tabular}

virus garantizará la eficacia de las medidas propuestas tendientes a su eliminación, planificada en una tercera etapa por SENASA. Durante el año 2010, y como primera etapa del plan, se realizó el primer relevamiento nacional de la EA teniendo como objetivos: estimar la prevalencia de establecimientos infectados, de cerdas en edad de reproducción infectadas y de cerdos de la categoría crecimiento-terminación infectados. Los resultados y los análisis epidemiológicos evidenciaron una prevalencia de $19,1 \%$ de predios infectados y $9 \%$ de cerdas infectadas. En ambos casos se evidencia una gran diferencia entre estratos poblacionales, siendo el más afectado el estrato con menor cantidad de madres (SENASA, 2015).

Es evidente la importancia de realizar estudios continuos de vigilancia sanitaria de poblaciones de cerdos silvestres para conocer las probabilidades de ingreso de un agente patógeno a las explotaciones pecuarias, como así también para disminuir riesgos para las especies amenazadas de la fauna silvestre a las que potencialmente podría afectar.

Los resultados del presente trabajo contribuyen a la vigilancia sanitaria de Argentina y brinda antecedentes de interacción interinstitucional para su mantenimiento en el tiempo. La vigilancia en fauna silvestre es fundamental para el mantenimiento y la documentación del status sanitario nacional. El impacto económico real o potencial de algunas de las enfermedades estudiadas en este trabajo puede ser muy grande y debe ser tenido en cuenta por los entes oficiales relacionados con la sanidad animal y la salud pública de nuestro país. 
En relación con la población de jabalíes o cerdos silvestres, el contacto de éstos con explotaciones de cerdos domésticos, como así también su utilización para el consumo de su carne, hace que sea relevante la incorporación de la misma a los planes nacionales de control de enfermedades y de esta manera controlar la aparición de nuevas enfermedades. Dicha población es considerada como de riesgo potencial debido a que está en contacto directo con otras poblaciones de especies domésticas y especies silvestres protegidas, como así también por ser fuente de alimento de pobladores rurales. Ambas características la convierten en una población que puede diseminar patógenos a otros animales y también a los humanos.

En conclusión, resulta evidente la importancia de realizar estudios continuos de vigilancia sanitaria de poblaciones de cerdos silvestres para conocer las probabilidades de ingreso de un agente patógeno a las explotaciones pecuarias, como así también para disminuir riesgos para las especies amenazadas de la fauna silvestre y para la salud pública. Una mejora y sistematización de la vigilancia, logrando interacción interdisciplinaria e interinstitucional, permitirá una mayor comprensión de los fenómenos naturales y no naturales que inciden sobre la ecología y el potencial epidémico de estas enfermedades.

\section{Agradecimientos}

Los autores agradecen la colaboración de Andrea Antonuci, Rodrigo Balzano, Graciela Cadenazzi, Rosa Debenedetti, Gabriela Maddonni, Celia Maidana, Ana Nicola, Andrea Pedemonte, Alejandro Pérez, Jessica Petrakovsky, Carlos Rodríguez, Marcos Suarez, Ricardo Veneroni, Carlos Zenobi y el resto del personal del Laboratorio Animal de SENASA. Este trabajo fue parcialmente financiado por el Servicio Nacional de Sanidad y Calidad Agroalimentaria de la República Argentina a través del Premio SENASA a la investigación, transferencia y comunicación de la sanidad, la inocuidad y la calidad agroalimentaria otorgado al proyecto: "Vigilancia epidemiológica en poblaciones de cerdos silvestres (Sus scrofa) implicancias para la salud pública, la producción animal y la conservación de la biodiversidad." y por un subsidio a la investigación otorgado por la Universidad Nacional Arturo Jauretche.

\section{Conflicto de intereses}

Todos los autores declaran que no existe conflicto de intereses, incluyendo las relaciones financieras, personales o de otro tipo con otras personas u organizaciones que pudieran influir de manera inapropiada en el trabajo.

\section{Bibliografía}

Al Dahouk S, Nockler K, Tomaso H, Splettstoesser WD, Jungersen G, Riber U, Petry T, Hoffmann D, Scholz HC, Hensel A, Neubauer H. 2005. Seroprevalence of brucellosis, tularemia, and yersiniosis in wild boars (Sus scrofa) from north-eastern Germany. Journal of Veterinary Medicine. Series B. 52(10):444-55.
Asociación Argentina de Veterinarios de Laboratorios de Diagnóstico (AAVLD). 1994. Manual de Leptospirosis. Buenos Aires.

Bharti A, Nally JE, Ricaldi JN, Matthias MA, Diaz MM, Lovett MA, Levett PN, Gilman RH, Wilig MR, Gotuzzo E, Vinetz JM. 2003. Leptospirosis: a zoonotic disease of global importance. The Lancet. Infectious Diseases. 3:757-71.

Carpinetti B. 1998. Spatio-temporal variation in a pampas deer (Ozotocerus bezoarticus celer) population: influence of habitat structure and sympatric ungulates. Tesis de Master Science, Durrell Institute of Conservation and Ecology, University of Kent at Canterbury, United Kingdom.

Carpinetti B, Castresana G, Rojas P, Grant J, Marcos A, Monterubbianesi M, Borrás P. 2014. Vigilancia epidemiológica en poblaciones de cerdos silvestres (Sus scrofa). Implicancias para la salud pública, la producción animal y la conservación de la biodiversidad. SNS. 6:67-76.

Corn J, Stallknecht D, Mechlin NM, Page Luttrell M, Fischer JR. 2004. Persistence of pseudorabies virus in feral swine populations. Journal of Wildlife Diseases. 40:307-10.

Crosby AW. 1986. Ecological imperialism: the biological expansion of Europe, 900-1900. New York, Cambridge University Press.

Daciuk J. 1978. Estado actual de las especies de mamíferos introducidos en la Subregión Araucana (República Argentina) y grado de coacción ejercido en algunos ecosistemas surcordilleranos. Anales de Parques Nacionales. 14:105-30.

Ebani VV, Cerri D, Poli A, Andreani E. 2003. Prevalence of leptospira and brucella antibodies in wild boars (Sus scrofa) in Tuscany, Italy. Journal of Wildlife Diseases. 39:718-22.

Giménez-Dixon M, 1991. Estimación de parámetros poblacionales del venado de las Pampas (Ozotoceros bezoarticus celer, Cabrera 1943 -Cervidae-) en la costa de la Bahía Samborombón (provincia de Buenos Aires) a partir de datos obtenidos mediante censos aéreos. Tesis de Doctorado en Ciencias Naturales, Facultad de Ciencias Naturales, Universidad Nacional de La Plata, Argentina.

Gresham CS, Gresham CA, Duffy MJ, Faulkner CT, Patton S. 2002. Increased prevalence of Brucella suis and pseudorabies virus antibodies in adults of an isolated feral swine population in coastal South Carolina. Journal of Wildlife Diseases. 38:653-6. doi:10.7589/0090-3558-38.3.653

Hudson PJ, Rizzoli A, Grenfell BT, Heesterbeek H, Dobson AP. 2002. The ecology of wildlife diseases. Oxford, Oxford University Press.

Jansen A, Luge E, Guerra B, Wittschen P, Gruber AD, Loddenkemper C, Schneider T, Lierz M, Ehlert D, Appel B, Stark K,Nöckler K. 2007. Leptospirosis in Urban Wild Boars, Berlin, Germany. Emerging Infectious Disease. 2007 May; 13(5): 739-742. doi: 10.3201/eid1305.061302

Jones K, Patel N, Levy M, Storeygard A, Balk D, Gittleman J, Daszak P. 2008. Global trends in emerging infectious diseases. Nature. 451:990-3.

Kern B, Depner KR, Leitz W, Rott M, Thalheim S, Nitschke B, Plagemann R, Liess B. 1999. Incidence of classical swine fever (CSF) in wild boar in a densely populated area indicating CSF virus persistence as a mechanism for virus perpetuation. Journal of Veterinary Medicine. Series B. 46:63-7.

Lari A, Lorenzi D, Nigrelli D, Brocchi E, Faccini S, Poli A. 2006. Pseudorabies virus in European wild boar from Central 
Italy. Journal of Wildlife Diseases. 42:319-24.

Mason RJ, Fleming PJ, Smythe LD, Dohnt MF, Norris MA, Symonds ML. 1998. Leptospira interrogans antibodies in feral pigs from New South Wales. Journal of Wildlife Diseases. 34:738-43.

Meng XJ, Lindsay DS, Sriranganathan N. 2009. Wild boars as sources for infectious diseases in livestock and humans. Philosophical transactions- Royal Society. Biological science. 364:2697-707.

Merino ML, Carpinetti B. 2003. Feral pig Sus scrofa population estimates in Bahía Samborombón conservation area, Buenos Aires Province, Argentina. Mastozoología Neotropical. 10(2):269-75.

Merino M, Vila A, Serret A. 1993. Relevamiento biológico de la Bahía Samborombón, provincia de Buenos Aires. Boletín Técnico Fundación Vida Silvestre Argentina. № 16.

Müller T, Teuffert J, Ziedler K, Possardt C, Kramer M, Staubach C, Conraths FJ. 1998. Pseudorabies in the European wild boar from eastern Germany. Journal of Wildlife Diseases. 34: 251-8.

Naranjo V, Gortazar C ,Vicente J, de la Fuente J. 2008. Evidence of the role of European wild boar as a reservoir of Mycobacterium tuberculosis complex. Veterinary Microbiology. 127(1-2): 1-9

Navas JA. 1987. Los vertebrados exóticos introducidos en la Argentina. Revista del Museo Argentino de Ciencias Naturales. 14 (2):7-38.

Nishi JS, Shury T, Elkin BT. 2006. Wildlife reservoirs for bovine tuberculosis (Mycobacterium bovis) in Canada: strategies for management and research. Veterinary Microbiology.112:325-38.

Paes R de C, Fonseca AA, Monteiro LA, Jardim GC, Piovezan U, Herrera HM, Mauro RA, Vieira-da-Motta O. 2013. Serological and molecular investigation of the prevalence of Aujeszky's disease in feral swine (Sus scrofa) in the subregions of the Pantanal wetland, Brazil. Veterinary Microbiology. 165:448-54. doi: 10.1016/j.vetmic.2013.03.028.

OIE. 2015. Manual de las pruebas de diagnóstico y de las vacunas para los animales terrestres. [ONLINE] Disponible en: http://www.oie.int/es/normas-internacionales/manualterrestre/acceso-en-linea/ [Consultado 10/10/2016].

Petrakovsky J, Tinao J, Esteves J. 2013. Leptospirosis porcina: prevalencia serológica en establecimientos productores de la República Argentina. Revista MVZ Córdoba. 18(1):3282-7.

Petrakovsky J, Carpinetti B, Antonucci A. 2016. Prevalencia serológica de Leptospira spp. en cerdos silvestres (Sus scrofa) en Bahía Samborombón, provincia de Buenos Aires, República Argentina, en el periodo 2013-2015. Salud y Tecnología Veterinaria. 3(1):23-7

Serena MS, Artuso MC, Pérez A, Echeverría MG, Laksman Y, Arocena G, Pereyra D, Escobar E, Sanguinetti HR, Zenobi $C$, Suárez MF, Debenedetti R, Marcos A, Borrás P, Castresana G, Rojas P, Monterubbianesi M, Grant J, Carpinetti B. 2015. Vigilancia epidemiológica de las principales virosis que afectan a las poblaciones de cerdos silvestres (Sus scrofa) en distintas zonas de la República Argentina. XI Congreso Argentino de Virología, CABA, Argentina, pp. 114-115.

van der Leek ML, Becker HN, Humphrey P, Adams CL, Belden RC, Frankenberger WB, Nicoletti PL. 1993. Prevalence of Brucella spp. antibodies in feral swine in Florida. Journal of Wildlife Diseases. 29:410-5.

Vengust G, Valencak Z, Bidovec A. 2006. A serological survey of selected pathogens in wild boar in Slovenia. Journal of Veterinary Medicine. Series B. 53:24-7.

SENASA. 2015. Enfermedad de Aujeszky. [ONLINE] Disponible en: (http://www.senasa.gov.ar/cadena-animal/porcinos/ produccion-primaria/sanidad-animal/enfermedades-y-estrasani/enfermedad-de-aujeszky-ea) [Consultado 10/10/2016].

Zupancic Z, Jukik B, Lojkik B, Jemers I, Starecina V. 2002. Prevalence of antibodies to classical swine fever, Aujeszky's disease, porcine reproductive and respiratory syndrome, and bovine viral diarrhoea viruses in wild boars in Croatia. Journal of Veterinary Medicine. Series B. 49:253-6. 